\title{
Clinical Outcomes and Quality of Life in Patients with Stanford Type B Aortic Dissection after Endovascular Repair
}

\author{
Yonghua Bi, MD, PhD,${ }^{1}$ Hongmei Chen, MD, ${ }^{2}$ Zepeng Yu, MD,${ }^{1}$ Jianzhuang Ren, MD, PhD, \\ Xinwei $\mathrm{Han}, \mathrm{MD}, \mathrm{PhD}^{1}$ \\ ${ }^{1}$ Department of Interventional Radiology, The First Affiliated Hospital of Zhengzhou University, Zhengzhou, China; \\ ${ }^{2}$ Department of Ultrasound, Zhengzhou Central Hospital Affiliated to Zhengzhou University, Zhengzhou, China
}

\section{ABSTRACT}

Background: Aortic dissection is a severe and life-threatening disease that is usually linked with numerous possible complications. Stanford type B aortic dissection patients often choose endovascular repair because of its mini-invasiveness and the possibility of quick recovery. This study is concerned with medial-term outcome and quality of life (QoL) in patients with Stanford type B aortic dissection after endovascular repair.

Methods: From January 2014 until July 2016, 40 patients with Stanford type B aortic dissection received an endovascular repair. Of the total number of patients, 35 were males $(87.5 \%)$ and 5 were females $(12.5 \%)$, mean aged $80.9 \pm 14.1$ years. The Medical Outcomes Study-Short Form-36 (MOS SF-36) was used to assess the QoL preoperatively and after endovascular repair. The first follow-up (FU) of MOS SF-36 questionnaire (FU1) was obtained within $3.9 \pm 0.3$ months after repair; and the second (FU2), $25.6 \pm 6.5$ months thereafter.

Results: None of patient died during the observational period, and one patient was lost at FU. The MOS SF-36 showed the best-scoring domain was "Role emotion," with "Vitality" and "Mental health" also scoring well preoperatively. Except for "Role emotion" and "Mental health," all remaining domains were significantly improved both at FU1 and FU2.

Conclusions: Endovascular repair in patients with Stanford type $\mathrm{B}$ aortic dissection enables excellent clinical outcomes and QoL.

\section{INTRODUCTION}

Aortic dissection is a severe and life-threatening event of sudden onset and is often linked with numerous possible complications. Operating on the thoracic aorta is challenging and associated with high mortality and morbidity

Received March 7, 2018; accepted May 14, 2018.

Y.B. and H.C. contributed equally and share as co-first authors.

Correspondence: Xinwei Han, MD, PhD, Professor of Department of Interventional Radiology, The First Affiliated Hospital of Zhengzhou University, No.1, East fian She Road, Zhengzhou 450052, China; +86-371-6686-2162; fax: +86-371-6686-2162 (e-mail: dreamweaver08@126.com).
[Jarral 2016]. Stanford type B aortic dissection patients more and more choose endovascular repair because of its mini-invasiveness and the possibility of quick recovery. Although many previous studies have been conducted to explore the clinical outcomes, survival of patients, and technical aspects of endovascular repair, little attention has been paid to the quality of life (QoL) assessment of patients after endovascular operations. Even though endovascular treatment is less invasive and patients recover more quickly, the impact of other factors, including the need for routine postprocedure CT scanning or the possible need for second intervention, on patients' QoL has not been clearly elucidated yet. There are only a few published studies dealing with patients' QoL after endovascular repair [Santini 2006; Endlich 2016]; however, postoperative QoL comparing physical and psychological status for patients with Stanford type B aortic dissection is rarely considered in this view. This study aims to evaluate the midterm outcome and QoL in patients with Stanford type $\mathrm{B}$ aortic dissection after endovascular repair.
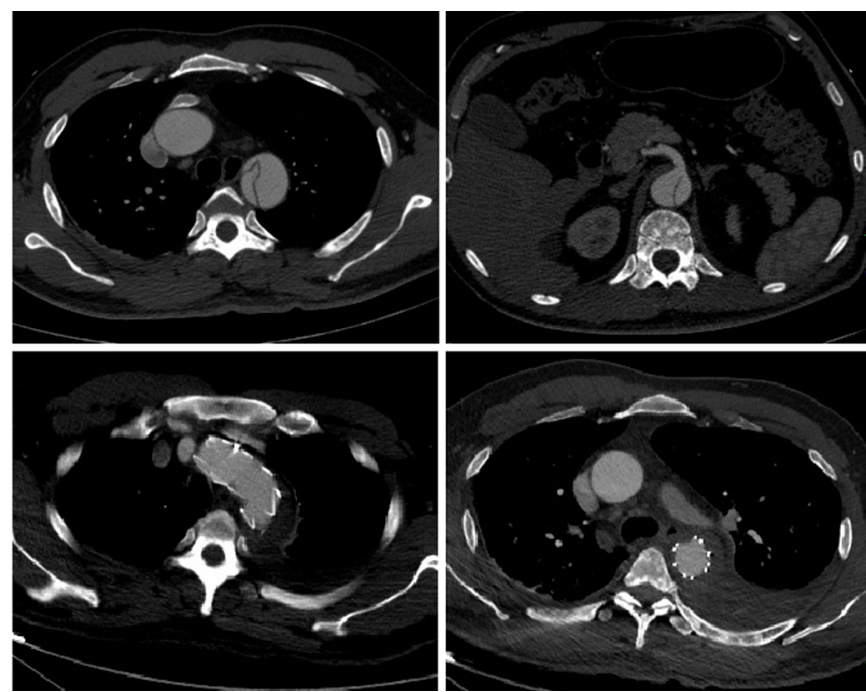

Figure 1. CT scanning before repair and during follow-up. Aortic dissection was shown in preoperative imaging (top pictures); covered stent was shown after intervention (bottom pictures). $<<$ At first proof, please confirm that Fig. 1 is showing what you mention in the figure legend. $>$ 
Table 1. Demographics of Patients with Stanford Type B Aortic Dissection

\begin{tabular}{|c|c|c|c|}
\hline Variables & No. $(\%)$ & Mean \pm SD & Range \\
\hline Patients & 40 & & \\
\hline Gender, male & $35(87.5 \%)$ & & \\
\hline Age, mean (years) & & $80.9 \pm 14.1$ & $28-81$ \\
\hline Type, typical 《<Clarify “Type, typical" »> & $35(87.5 \%)$ & & \\
\hline \multicolumn{4}{|c|}{ Medical history before acute dissection } \\
\hline Myocardial ischemia & $2(5 \%)$ & & \\
\hline Aortic surgery & $2(5 \%)$ & & \\
\hline Diabetes mellitus & $1(2.5 \%)$ & & \\
\hline Peripheral vascular disease & $2(5 \%)$ & & \\
\hline Neurologic dysfunction & $1(2.5 \%)$ & & \\
\hline Arterial hypertension & $23(57.5 \%)$ & & \\
\hline Smoking & $21(52.5 \%)$ & & \\
\hline Marfan syndrome & $1(2.5 \%)$ & & \\
\hline \multicolumn{4}{|l|}{ Symptoms } \\
\hline Pain & $32(80 \%)$ & & \\
\hline Chest distress & $4(10 \%)$ & & \\
\hline Syncope & $1(2.5 \%)$ & & \\
\hline \multicolumn{4}{|l|}{ Course of disease } \\
\hline Hours, mean & $18(45 \%)$ & $7.5 \pm 4.0$ & $3-19$ \\
\hline Days <<Add “mean"?>> & $18(45 \%)$ & $5.1 \pm 6.4$ & $1-25$ \\
\hline Months <<Add “mean"? >> & $4(10 \%)$ & $6.0 \pm 10.0$ & $1-21$ \\
\hline
\end{tabular}

\section{MATERIALS AND METHODS}

\section{Patient Selection}

This study was approved by the Ethics Committee of Zhengzhou University. From January 2014 until July 2016, 40 patients with Stanford type B aortic dissection received an endovascular repair. The Medical Outcomes Study-Short Form-36 (MOS SF-36) was used to assess the QoL preoperatively and after endovascular repair. All patients underwent CT scanning (Figure 1) and were asked to answer MOS SF-36 questionnaires during routine follow-up (FU), and 1 was lost at FU.

\section{Questionnaires and Data Collection}

The MOS SF-36 questionnaire comprises 36 items and yields an 8-subscores profile of functional-health and wellbeing scores. Scores range from 0 to 100. A Chinese version of the standard MOS SF-36 questionnaire has been validated for a Chinese population [Song 1984]. Data set was acquired by using the MOS SF-36 QoL questionnaire. The first follow-up of MOS SF-36 questionnaire (FU1) was obtained within $3.9 \pm 0.3$ months after repair; the second (FU2), $25.6 \pm$ 6.5 months thereafter. The MOS SF-36 FU was gathered by telephone calls for some of the patients who could not show up for their FU because of age or refusal to come to the clinic.

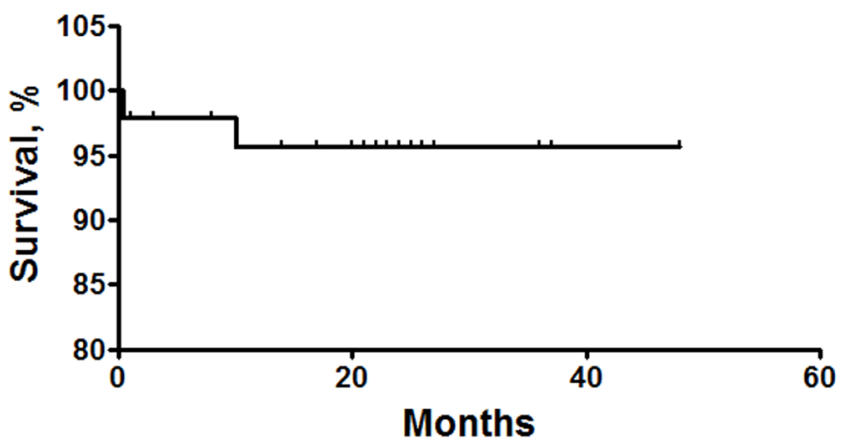

Figure 2. Survival rates. The 1-year and 4-year survival rates were both $95.7 \%$.

Patients who were unable to fill in the questionnaires themselves, for example, because of dementia, were excluded from this study.

\section{Statistical Analysis}

The statistical analysis was performed with Prism 5.0 software (GraphPad Software, La Jolla, CA, USA). Statistics are summarized as mean value, and one-way analysis of variance was used for continuous variables. Mean values are given with standard deviation (SD) in this form: mean \pm SD. Measures of dispersion are summarized as SD or range. Chi-square test was used to test percentage scoring. For all tests, bilateral significance was tested and accepted as significant with $P<.05$.

\section{RESULTS}

\section{Demographics}

Table 1 displays the clinical profiles. Of the total number of patients, there were 35 males $(87.5 \%)$ and 5 females (12.5\%), mean aged $80.9 \pm 14.1$ years. There was $1(2.5 \%)$ patient with Marfan syndrome. Of the patients, $23(57.5 \%)$ had arterial hypertension, $21(52.5 \%)$ were smokers, $2(5.0 \%)$ showed peripheral vascular disease, and $2(5.0 \%)$ had history of aortic surgery. Furthermore, there were $1(2.5 \%)$ with neurologic dysfunction, $1(2.5 \%)$ with insulin-dependent diabetes mellitus type 2 , and $2(5.0 \%)$ with previous myocardial ischemia.

\section{Clinical Presentation}

Thirty-five $(87.5 \%)$ patients were of a complicated type of aortic dissection, and $5(12.5 \%)$ were of an uncomplicated type, including aortic ulcer and aortic intramural hematoma. Preoperatively, 32 (80\%) patients complained of pain (thoracalgia, dorsodynia, osphyalgia, and abdominal pain), 4 (10\%) showed chest distress, and $1(2.5 \%)$ had a syncope. For the course of the disease, 18 (45\%) of all patients were admitted to the hospital within hours or days, with mean course of 7.5 \pm 4.0 hours and $5.1 \pm 6.4$ days. Four $(10 \%)$ patients showed a course of months on admission, with a mean of $6.0 \pm 10.0$ months (Table 1). 
Table 2. Results of the MOS SF-36 Questionnaire

\begin{tabular}{|c|c|c|c|c|c|}
\hline Physical functioning & $35.77 \pm 27.59$ & $86.30 \pm 10.25$ & $89.04 \pm 10.20$ & $P<.0001$ & $P<.0001$ \\
\hline Bodily pain & $33.67 \pm 32.88$ & $91.63 \pm 14.85$ & $92.12 \pm 14.38$ & $P<.0001$ & $P<.0001$ \\
\hline General health & $55.51 \pm 22.50$ & $81.85 \pm 9.00$ & $80.38 \pm 13.78$ & $P<.0001$ & $P<.0001$ \\
\hline Social functioning & $58.67 \pm 24.34$ & $90.22 \pm 9.32$ & $93.23 \pm 7.67$ & $P<.0001$ & $P<.0001$ \\
\hline Role emotion & $86.33 \pm 30.33$ & $81.56 \pm 26.68$ & $77.08 \pm 24.48$ & ns & ns \\
\hline Mental health & $75.69 \pm 14.20$ & $80.44 \pm 7.05$ & $81.85 \pm 9.35$ & ns & ns \\
\hline
\end{tabular}

Pre, preoperation; FU, follow-up; ns, not significant.

\section{Quality of Life}

The distributions of MOS SF-36 domain scores are shown in Table 2. MOS SF-36 observation showed the best-scoring domain was "Role emotion," with a mean of $86.33 \pm 30.33$, before endovascular repair. The "Vitality" and "Mental health" also showed scoring well preoperatively, with mean of $74.74 \pm 19.46$ and $75.69 \pm 14.20$, respectively. The worstscoring domain was "Role physical" $(21.15 \pm 36.52)$ before operation. Except for "Role emotion" and "Mental health," all remaining domains were significantly improved at both FU1 and FU2. There was no significant change between FU1 and FU2. Good score \%, percentage scoring $\geq 80$ points, is shown in Table 3. All domains, but "Role emotion," "Mental health," and "Vitality," were significantly improved during FU; the majority of patients said that their healthy condition was significant improved when compared with one year ago $(P<.0001)$.

\section{Operative Techniques}

As shown in Table 4, endovascular procedures consisted of sole endovascular repair in $32(80 \%)$ patients, endovascular repair combined with bypass of the left subclavian artery and the left common carotid artery bypass in $4(10 \%)$ cases. Endovascular repair with chimney stenting of left subclavian artery was performed in $3(7.5 \%)$ cases, and endovascular repair combined with left femoral artery stenting in $1(2.5 \%)$ case. Thirtyfour $(85 \%)$ patients were under general anesthesia, and $6(15 \%)$ cases underwent repair under local anesthesia combined with regional block anesthesia. The covered aortic stents were introduced by the right femoral artery in $34(85 \%)$ cases, and $6(15 \%)$ patients received them via left femoral artery access. Brachial artery puncture was used for angiography and blood pressure monitoring during repair. Two patients did not receive brachial artery puncture, and angiography was performed via femoral access in this circumstance.

\section{Complications}

None of patient died during the observational period, and 1 patient was lost at FU. Perioperative endoleak was the most common complication, shown in 7 patients (17.5\%); 3 had type I endoleak; 1 had type II endoleak; and the others had type III endoleak; all endoleaks ceased without treatment during repair operation and FU. During the FU, none of patients died because of complications, such as rupture of aorta, low cardiac output, multiple organ failure (MOF), or bleeding. There was a variety of complications; of the patients, 2 (5\%) developed left hand numbness with no disability; 2 (5\%) developed postoperative delirium and was transferred to ICU. One $(2.5 \%)$ suffered from right hemothorax; 1 (2.5\%) developed hemopericardium; and 2 (5\%) suffered from pleural effusion; these 2 patients were in need of pleural drainage. Open operational repair was performed in $1(2.5 \%)$ case because of reverse tear of aortic dissection 11 days after endovascular repair (Table 4).

\section{Follow-up}

All patients were followed up, except for 1 patient lost at FU. One patient died of aorta rupture after successful repair. Except 1 perioperative death, none of the patients died after repair because of complications. One patient showed recurrent dissection 2 years later and underwent a second endovascular repair. The 1-year and 4-year survival rates were both 95.7\% (Figure 2).

\section{DISCUSSION}

Acute aortic dissection is a life-threatening disease, in which the aorta may enlarge, rupture, or suffer new dissections and cause sudden death potentially. Operating on the thoracic aorta is challenging and associated with high mortality and morbidity [Jarral 2016]. The introduction of endovascular repair into clinical practice has decreased perioperative mortality and reduced complications. The number and percentage of postoperative complications are very low, with an excellent survival rate during FU.

The MOS SF-36 questionnaire was used as a generic questionnaire, which is a well accepted and highly validated tool with a large evaluated data set. Measurement of QoL using the MOS SF-36 questionnaire after thoracic aortic surgery was a point of interest in various papers [Olsson 1999; Stalder 2007; 
Table 3. Good score of the MOS SF-36 Questionnaire

\begin{tabular}{lcccc}
\hline & Pre & FU1 & FU2 & $P$ \\
\hline Physical functioning & 7.7 & 77.8 & 84.6 & $<.0001$ \\
Role physical & 12.8 & 44.4 & 57.7 & $<.0001$ \\
Bodily pain & 15.4 & 81.5 & 88.5 & $<.05$ \\
General health & 20.5 & 74.1 & 65.4 & $<.0001$ \\
Vitality & 61.5 & 74.1 & 80.8 & $\mathrm{~ns}$ \\
Social functioning & 10.3 & 88.9 & 96.2 & $<.0001$ \\
Role emotion & 79.5 & 59.3 & 54.3 & $\mathrm{~ns}$ \\
Mental health & 56.4 & 70.4 & 76.9 & $\mathrm{~ns}$ \\
\hline
\end{tabular}

Good score $\%$ is the percentage scoring $\geq 80$. Pre, preoperation; FU, followup; ns, not significant.

$<<$ Confirm expansions added in the footnote. $>>$

$<<$ See main text for query on "Good score \%"; adjust title and footnote if needed.>

Lohse 2009]. The MOS SF-36 questionnaire was used for patients with acute aortic dissection type A who received open repair [Santini 2006; Endlich 2016] and for patients with type A acute aortic dissection after aortic surgery [Franke 2010], as well as for patients with abdominal aortic aneurysm repair [Kisis 2012; Lottman 2004]. Two studies examined QoL following endovascular repair with and without coverage of the left subclavian artery [Klocker 2014; McBride 2015], and one study investigated QoL by the SWED-QUAL questionnaire for patients with conservatively treated acute type B aortic dissection [Winnerkvist 2006]. There are few studies of QoL for Stanford type B aortic dissection patients, so this study mainly focuses on the QoL after endovascular repair.

In our study, the "Role physical" components, "Bodily pain" components, and "Physical functioning" components received the lowest scores preoperatively; many patients were restricted to daily activities, and their "Role physical" and "Physical functioning" dramatically deteriorated. Patients worried about being unable to carry out normal activities in work and daily life. It is not convenient that the patients must rest strictly in bed with ECG and blood pressure monitoring before and about 2 weeks after endovascular repair. However, the "Role emotion" and "Mental health" components scored well, which were not significantly changed after endovascular repair. Our studies show very few patients scoring $\geq 80 \%$ in all domains except for "Role emotion," "Mental health," and "Vitality" before operation; however, these domains significantly improved after endovascular repair both at FU1 and FU2; this reveals that endovascular repair does seem to have an overall effect on QoL. Interestingly, there was no significant difference between FU1 and FU2 in all domains, which indicates that patients who underwent endovascular repair recovered quickly and that their QoL significantly improved within $3.9 \pm 0.3$ months after repair (FU1).

From our knowledge, to date, there is no 2-point postoperative FU study concerning QoL in Stanford type B aortic
Table 4. Operative Techniques of Patients with Stanford Type B Aortic Dissection

\begin{tabular}{lc}
\hline & No. $(\%)$ \\
\hline $\begin{array}{l}\text { Type of endovascular repair } \\
\text { Sole endovascular repair <<For “Sole endovascular } \\
\text { repair," do you mean “Only endovascular repair”? If } \\
\text { so, please make a change.>> }\end{array}$ & $32(80 \%)$ \\
$\quad$ Endovascular repair + Bypass & \\
Endovascular repair + Chimney stenting & $4(10 \%)$ \\
Endovascular repair + Left femoral artery stenting & $3(7.5 \%)$ \\
Anesthesia & $1(2.5 \%)$ \\
General anesthesia & \\
Local anesthesia+ Regional block anesthesia & $34(85 \%)$ \\
Complications & $6(15 \%)$ \\
Endoleak & \\
Reverse tear of dissection & $7(17.5 \%)$ \\
Right hemothorax & $1(2.5 \%)$ \\
Hemopericardium & $1(2.5 \%)$ \\
Pleural effusion & $1(2.5 \%)$ \\
Left hand numbness & $2(5 \%)$ \\
Postoperative delirium & $2(5 \%)$ \\
$2(5 \%)$
\end{tabular}

dissection patients. In contrast to a single-point FU evaluation, which gives a snapshot, 2-point measurements allow for an estimation concerning the course of this chronic disease. Limitations of this study are predominantly caused by the single-center approach with a small sample size, which does not allow for prospective randomization. A registry for endovascular repair could produce more precise data on the QoL; further studies involving a larger patient population are needed.

In conclusion, this study demonstrates beneficial outcomes of QoL for patients with Stanford type B aortic dissection undergoing endovascular repair.

\section{ACKNOWLEDGMENTS}

This work was supported by the National Natural Science Foundation of China (grant no. 81501569).

The authors declare that there is no conflict of interest.

\section{REFERENCES}

Endlich M, Hamiko M, Gestrich C, et al. 2016. Long-term outcome and quality of life in aortic type A dissection survivors. Thorac Cardiovasc Surg 64:91-9.

Franke UF, Isecke A, Nagib R, et al. 2010. Quality of life after aortic root surgery: reimplantation technique versus composite replacement. Ann Thorac Surg 90:1869-75.

Jarral OA, Kidher E, Patel VM, Nguyen B, Pepper J, Athanasiou T. 2016. Quality of life after intervention on the thoracic aorta. Eur J Cardiothorac Surg 49:369-89. 
Kisis K, Krievins D, Naskovica K, et al. 2012. Quality of life after endovascular abdominal aortic aneurysm repair: Nellix sac-anchoring endoprosthesis versus open surgery. Medicina (Kaunas) 48:286-91.

Klocker J, Koell A, Erlmeier M, Goebel G, Jaschke W, Fraedrich G. 2014. Ischemia and functional status of the left arm and quality of life after left subclavian artery coverage during stent grafting of thoracic aortic diseases. J Vasc Surg 60:64-9.

Lohse F, Lang N, Schiller W, et al. 2009. Quality of life after replacement of the ascending aorta in patients with true aneurysms. Tex Heart Inst J 36:104-10.

Lottman PE, Laheij RJ, Cuypers PW, Bender M, Buth J. 2004. Healthrelated quality of life outcomes following elective open or endovascular AAA repair: a randomized controlled trial. J Endovasc Ther 11:323-9.

McBride CL, Dubose JJ, Miller CC 3rd, et al. 2015. Intentional left subclavian artery coverage during thoracic endovascular aortic repair for traumatic aortic injury. J Vasc Surg 61:73-9.

Olsson C, Thelin S. 1999. Quality of life in survivors of thoracic aortic surgery. Ann Thorac Surg 67:1262-7.

Santini F, Montalbano G, Messina A, et al. 2006. Survival and quality of life after repair of acute type A aortic dissection in patients aged 75 years and older justify intervention. Eur J Cardiothorac Surg 29:386-91.

Song YG, Chen GZ, Song YL. 1984. The free thigh flap: a new free flap concept based on the septocutaneous artery. Br J Plast Surg 37:149-59.

Stalder M, Staffelbach S, Immer FF, et al. 2007. Aortic root replacement does not affect outcome and quality of life. Ann Thorac Surg 84:775-80; discussion 780-1.

Winnerkvist A, Brorsson B, Radegran K. 2006. Quality of life in patients with chronic type B aortic dissection. Eur J Vasc Endovasc Surg 32:34-7. 